\title{
Gribov copies, lattice QCD and the gluon propagator
}

\author{
P.J. Silva, O. Oliveira \\ Centro de Física Computacional, Departamento de Física, Universidade de Coimbra, \\ 3004-516 Coimbra, Portugal
}

Received 26 March 2004; accepted 23 April 2004

\begin{abstract}
We address the problem of Gribov copies in lattice QCD. The gluon propagator is computed, in the Landau gauge, using $302(\beta=5.8) 12^{4} S U(3)$ configurations gauge fixed to different copies. The results of the simulation shows that: (i) the effect of Gribov copies is small (less than 10\%); (ii) Gribov copies change essentially the lowest momenta components $(q<2.6 \mathrm{GeV})$; (iii) within the statistical accuracy of our simulation, the effect of Gribov copies is resolved if statistical errors are multiplied by a factor of two or three. Moreover, when modelling the gluon propagator, different sets of Gribov copies produce different sets of parameters not, necessarily, compatible within one standard deviation. Finally, our data supports a gluon propagator which, for large momenta, behaves like a massive gluon propagator with a mass of $1.1 \mathrm{GeV}$.
\end{abstract}

(c) 2004 Elsevier B.V. All rights reserved.

PACS: 12.38.G; 11.15.H

Keywords: Lattice QCD; Landau gauge; Gauge fixing; Gribov copies; Gluon propagator; Gluon mass

\section{Introduction and motivation}

Quantum chromodynamics (QCD) is the theory that describes the interaction between quarks and gluons. The definition of the QCD generating functional à la Faddeev-Popov [1-3] requires a choice of a gauge condition, uniquely satisfied in each gauge orbit, i.e., on each set of fields related by a gauge transformation. For the Landau, the Coulomb gauge and for small field amplitudes, the gauge condition is uniquely satisfied in each gauge orbit.

E-mail addresses: psilva@teor.fis.uc.pt (P.J. Silva), orlando@teor.fis.uc.pt (O. Oliveira). 
However, if large field amplitudes are involved, the gauge fixing condition has multiple solutions in each gauge orbit [4,5], the Gribov copies.

Gribov copies appear when large field amplitudes are involved and rise the question of how to define the generating functional for the nonperturbative regime of quantum chromodynamics. Moreover, in [6] it was proved that it is not possible to find a local continuous and unambiguous gauge fixing condition for any $S U(N)$ gauge theory defined on the manifold $S_{4}$. A similar result for the four-torus was obtained in [7].

For the continuum formulation of QCD, Zwanziger argued in [8] that the Landau gauge Faddeev-Popov formula

$$
\delta(\partial A) \operatorname{det}[-\partial \cdot D(A)] \exp \left[-S_{\mathrm{YM}}(A)\right],
$$

restricted to the region where the Faddeev-Popov operator is positive definite $-\partial \cdot D(A)>$ 0 , the Gribov region $\Omega$, provides an exact nonperturbative quantization for QCD. This result helps to eliminate some theoretical questions about the investigations of QCD using Dyson-Schwinger equations (DSE). Nevertheless, in what concerns the nonperturbative regime of QCD, being unable to solve exactly the DSE, the results of such studies should be compared to lattice results. In this way one can test the validity of the approximations and ansatz used to solve the DSE and, simultaneously, the lattice algorithms.

The formulation of gauge theories on the lattice does not require gauge fixing. As long as one is interested only on gauge invariant operators, the lattice calculation is not plagued with the problem of Gribov copies. However, the investigation of the Green's functions of the fundamental fields, such as the gluon, ghost and fermion propagators, implies the choice of a gauge. On the lattice, typically, a simulation begins by generating a number of thermalized gauge configurations. In order to compute, for example, the propagators, each configuration is then rotated to satisfy a given gauge fixing condition. Finally, the propagator is computed using these rotated configurations. For the Landau gauge, gauge fixing is implemented by computing a maximum of a given function defined on the gauge orbits. Now, the problem of the Gribov copies is due to the several maxima of the maximizing function. The first observations and studies of lattice Gribov copies were done long ago [9-14]. However, how the choice of Gribov copies changes the correlation functions is not yet clear.

On the continuum formulation, Gribov [4] studied $S U$ (2) gauge theory. His proposal to solve the problem of the different copies was to restrict the functional integration space to the so-called Gribov region $\Omega$. The gluon propagator computed by functional integrating the gluon fields over $\Omega$ does not show the usual perturbative $1 / q^{2}$ behavior but, instead, $q^{2} /\left(q^{4}+M^{4}\right)$, with $M$ being a mass scale which measures the volume of $\Omega$. Note that the two propagators agree for the high energy regime.

On the lattice, there was a number of studies about Gribov copies and different observables in various gauges. In this paper we will be mainly concerned about the gluon propagator computed in the Landau gauge. For a general discussion about lattice Gribov copies see, for example, [15] and references therein. For the $S U(2)$ group, the gauge and ghost propagators versus Gribov copies were studied in $[16,17]$. The authors claim that the gluon propagator is not sensible to Gribov copies in the weak coupling regime. ${ }^{1}$ For 
the ghost propagator, the simulations performed by the first author shows that, close to the continuum, the propagator is again not sensible to Gribov copies. In the second study, it is claimed a reduction of $6 \%$ for the central value of the smallest momenta ghost propagator and a reduction of $4 \%$ on the Kugo-Ojima parameter. ${ }^{2}$ The $S U(2)$ simulations suggest that the influence of Gribov copies is at the level of the simulation statistical error. For $S U(3)$ there is no systematic study but it is believed that the Gribov noise is contained within the statistical error of the Monte Carlo.

In this paper we study the pure gauge lattice QCD gluon propagator in the Landau gauge and try to understand the role of the Gribov copies. We compute the gluon propagator for 302 configurations, with $\beta=5.8$, for a lattice size of $12^{4}$. Our results show that, although being a small effect (less than 6-10\%), the Gribov copies change the lowest momenta components of the gluon propagator. This effect is illustrated fitting the gluon propagator and comparing the results for sets of configurations built from different copies. Gribov copies influence can go from a doubling of the statistical error, to the extreme case of changing the functional form of the propagator.

The paper is organized as follows. Section 2 sets the field definitions and notation used in this work. In Section 3, the Landau gauge is discussed, both on the continuum and in lattice QCD. Moreover, the algorithm used here is sketched. In Section 4, the results for the role of Gribov copies in the gluon propagator are reported. Finally, in Section 5 our results are discussed.

\section{Field definitions and notation}

In the lattice formulation of $\mathrm{QCD}$, the gluon fields $A_{\mu}^{a}$ are replaced by the links

$$
U_{\mu}(x)=e^{i a g_{0} A_{\mu}\left(x+a \hat{e}_{\mu} / 2\right)}+\mathcal{O}\left(a^{3}\right) \in S U(3),
$$

where $\hat{e}_{\mu}$ are unit vectors along $\mu$ direction. QCD is a gauge theory, therefore the fields related by gauge transformations

$$
U_{\mu}(x) \rightarrow g(x) U_{\mu}(x) g^{\dagger}\left(x+a \hat{e}_{\mu}\right), \quad g \in S U(3),
$$

are physically equivalent. The set of links related by gauge transformations to $U_{\mu}(x)$ is the orbit of $U_{\mu}(x)$.

The gluon field associated to a gauge configuration is given by

$$
A_{\mu}\left(x+a \hat{e}_{\mu} / 2\right)=\frac{1}{2 i g_{0}}\left[U_{\mu}(x)-U_{\mu}^{\dagger}(x)\right]-\frac{1}{6 i g_{0}} \operatorname{Tr}\left[U_{\mu}(x)-U_{\mu}^{\dagger}(x)\right]
$$

up to corrections of order $a^{2}$.

\footnotetext{
${ }^{1}$ Note that, in the strong coupling regime, Cucchieri is able to see differences on the propagator due to Gribov copies.

${ }^{2}$ See, also, [18].
} 
On the lattice, due to the periodic boundary conditions, the discrete momenta available are

$$
\hat{q}_{\mu}=\frac{2 \pi n_{\mu}}{a L_{\mu}}, \quad n_{\mu}=0,1, \ldots, L_{\mu}-1,
$$

where $L_{\mu}$ is the lattice length over direction $\mu$. The momentum space link is

$$
U_{\mu}(\hat{q})=\sum_{x} e^{-i \hat{q} x} U_{\mu}(x)
$$

and the momentum space gluon field

$$
\begin{aligned}
A_{\mu}(\hat{q}) & =\sum_{x} e^{-i \hat{q}\left(x+a \hat{e}_{\mu} / 2\right)} A_{\mu}\left(x+a \hat{e}_{\mu} / 2\right) \\
& =\frac{e^{-i \hat{q}_{\mu} a / 2}}{2 i g_{0}}\left\{\left[U_{\mu}(\hat{q})-U_{\mu}^{\dagger}(-\hat{q})\right]-\frac{1}{3} \operatorname{Tr}\left[U_{\mu}(\hat{q})-U_{\mu}^{\dagger}(-\hat{q})\right]\right\} .
\end{aligned}
$$

The gluon propagator is the gluon two point correlation function. The dimensionless lattice two point function is

$$
\left\langle A_{\mu}^{a}(\hat{q}) A_{\nu}^{b}\left(\hat{q}^{\prime}\right)\right\rangle=D_{\mu \nu}^{a b}(\hat{q}) V \delta\left(\hat{q}+\hat{q}^{\prime}\right) .
$$

On the continuum, the momentum space propagator in the Landau gauge is given by

$$
D_{\mu \nu}^{a b}(\hat{q})=\delta^{a b}\left(\delta_{\mu \nu}-\frac{q_{\mu} q_{\nu}}{q^{2}}\right) D\left(q^{2}\right) .
$$

Assuming that the deviations from the continuum are negligible, the lattice scalar function $D\left(q^{2}\right)$ can be computed directly from (9) as follows

$$
D\left(q^{2}\right)=\frac{2}{\left(N_{c}^{2}-1\right)\left(N_{d}-1\right) V} \sum_{\mu}\left\langle\operatorname{Tr}\left[A_{\mu}(\hat{q}) A_{\mu}(-\hat{q})\right]\right\rangle, \quad q \neq 0,
$$

and

$$
D(0)=\frac{2}{\left(N_{c}^{2}-1\right) N_{d} V} \sum_{\mu}\left\langle\operatorname{Tr}\left[A_{\mu}(\hat{q}) A_{\mu}(-\hat{q})\right]\right\rangle, \quad q=0,
$$

where

$$
q_{\mu}=\frac{2}{a} \sin \left(\frac{\hat{q}_{\mu} a}{2}\right),
$$

$N_{c}=3$ is the dimension of the group, $N_{d}=4$ the number of spacetime dimensions and $V$ is the lattice volume.

\section{The Landau gauge}

\subsection{The continuum Landau gauge}

On the continuum, the Landau gauge is defined by

$$
\partial_{\mu} A_{\mu}=0
$$


This condition defines the hyperplane of transverse configurations

$$
\Gamma \equiv\{A: \partial \cdot A=0\}
$$

It is well known [4] that $\Gamma$ includes more than one configuration from each gauge orbit. In order to try to solve the problem of the nonperturbative gauge fixing, Gribov suggested the use of additional conditions, namely the restriction of physical configurational space to the region

$$
\Omega \equiv\{A: \partial \cdot A=0, M[A] \geqslant 0\} \subset \Gamma,
$$

where $M[A] \equiv-\nabla \cdot D[A]$ is the Faddeev-Popov operator. However, $\Omega$ is not free of Gribov copies and does not provide a proper definition of physical configurations.

A suitable definition of the physical configurational space is given by the fundamental modular region $\Lambda \subset \Omega$, the set of the absolute minima of the functional

$$
F_{A}[g]=\int d^{4} x \sum_{\mu} \operatorname{Tr}\left[A_{\mu}^{g}(x) A_{\mu}^{g}(x)\right] .
$$

The fundamental modular region $\Lambda$ is a convex manifold [19] and each gauge orbit intersects the interior of $\Lambda$ only once [20,21], i.e., its interior consists of nondegenerate absolute minima. On the boundary $\partial \Lambda$ there are degenerate absolute minima, i.e., different boundary points are Gribov copies of each other [21-23]. The interior of $\Lambda$, the region of absolute minima of (16), identifies a region free of Gribov copies.

\subsection{The lattice Landau gauge}

On the lattice, the situation is similar to the continuum theory [24-26]. The interior of $\Lambda$ consists of nondegenerate absolute minima of the lattice version of (16) and Gribov copies can occur at the boundary $\partial \Lambda$. However, for a finite lattice, the boundary $\partial \Lambda$, where degenerate minima may occur, has zero measure and the presence of these minima can be ignored [25].

On the lattice, the Landau gauge is defined by maximizing the functional

$$
F_{U}[g]=C_{F} \sum_{x, \mu} \operatorname{Re}\left\{\operatorname{Tr}\left[g(x) U_{\mu}(x) g^{\dagger}(x+\hat{\mu})\right]\right\},
$$

where

$$
C_{F}=\frac{1}{N_{d} N_{c} V}
$$

is a normalization constant. Let $U_{\mu}$ be the configuration that maximizes $F_{U}[g]$ on a given gauge orbit. For configurations near $U_{\mu}$ on its gauge orbit, we have

$$
\begin{aligned}
F_{U}[1+i \omega(x)]= & F_{U}[1]+\frac{C_{F}}{4} \sum_{x, \mu} i \omega^{a}(x) \operatorname{Tr}\left[\lambda^{a}\left(U_{\mu}(x)-U_{\mu}(x-\hat{\mu})\right)\right. \\
& \left.-\lambda^{a}\left(U_{\mu}^{\dagger}(x)-U_{\mu}^{\dagger}(x-\hat{\mu})\right)\right],
\end{aligned}
$$


where $\lambda^{a}$ are the Gell-Mann matrices. By definition, $U_{\mu}$ is a stationary point of $F$, therefore

$$
\begin{aligned}
\frac{\partial F}{\partial \omega^{a}(x)}= & \frac{i C_{F}}{4} \sum_{\mu} \operatorname{Tr}\left[\lambda^{a}\left(U_{\mu}(x)-U_{\mu}(x-\hat{\mu})\right)\right. \\
& \left.-\lambda^{a}\left(U_{\mu}^{\dagger}(x)-U_{\mu}^{\dagger}(x-\hat{\mu})\right)\right]=0 .
\end{aligned}
$$

In terms of the gluon field, this condition reads

$$
\sum_{\mu} \operatorname{Tr}\left[\lambda^{a}\left(A_{\mu}(x+a \hat{\mu} / 2)-A_{\mu}(x-a \hat{\mu} / 2)\right)\right]+\mathcal{O}\left(a^{2}\right)=0,
$$

or

$$
\sum_{\mu} \partial_{\mu} A_{\mu}^{a}(x)+\mathcal{O}(a)=0,
$$

i.e., (20) is the lattice equivalent of the continuum Landau gauge condition. The lattice Faddeev-Popov operator $M(U)$ is given by the second derivative of (17).

Similarly to the continuum theory, on the lattice one defines the region of stationary points of (17)

$$
\Gamma \equiv\{U: \partial \cdot A(U)=0\},
$$

the Gribov's region $\Omega$ of the maxima of (17),

$$
\Omega \equiv\{U: \partial \cdot A(U)=0 \text { and } M(U) \geqslant 0\}
$$

and the fundamental modular region $\Lambda$ defined as the set of the absolute maxima of (17).

A proper definition of the lattice Landau gauge chooses from each gauge orbit, the configuration belonging to the interior of $\Lambda$.

\subsection{Gauge fixing algorithm}

On the lattice, gauge fixing is implemented by maximizing $F_{U}[g]$. In this work, the gauge fixing algorithm used is a Fourier accelerated steepest descent method (SD) as defined in [27]. In each iteration, the algorithm chooses

$$
g(x)=\exp \left[\hat{F}^{-1} \frac{\alpha}{2} \frac{p_{\max }^{2} a^{2}}{p^{2} a^{2}} \hat{F}\left(\sum_{v} \Delta_{-v}\left[U_{v}(x)-U_{\nu}^{\dagger}(x)\right]-\text { trace }\right)\right],
$$

where

$$
\Delta_{-v}\left(U_{\mu}(x)\right)=U_{\mu}\left(x-a \hat{e}_{\nu}\right)-U_{\mu}(x),
$$

$p^{2}$ are the eigenvalues of $\left(-\partial^{2}\right), a$ is the lattice spacing and $\hat{F}$ represents a fast Fourier transform (FFT). For the parameter $\alpha$ we use the value 0.08 [27]. For numerical purposes, it is enough to expand to first order the exponential in (25), followed by a reunitarization of $g(x)$.

On the gauge fixing process, the quality of the gauge fixing is measured by

$$
\theta=\frac{1}{V N_{c}} \sum_{x} \operatorname{Tr}\left[\Delta(x) \Delta^{\dagger}(x)\right]
$$


where

$$
\Delta(x)=\sum_{v}\left[U_{v}\left(x-a \hat{e}_{v}\right)-U_{v}^{\dagger}(x)-\text { h.c. }- \text { trace }\right]
$$

is the lattice version of $\partial_{\mu} A_{\mu}=0$.

\section{The gluon propagator}

In this work only pure gauge quenched configurations are considered. The Wilson action configurations were generated with version 6 of MILC code [28].

The function $F_{U}$ has many maxima-see, for example, [29]. In each gauge orbit, the different maxima are different configurations and, therefore, the gluon propagator changes according to the chosen set of maxima. In order to study such dependence, 302 gauge configurations were generated for a $12^{4}$ lattice and for $\beta=5.8$, using a combined update of 4 over-relaxed and 5 quasi-heat bath Cabbibo-Mariani updates, with a separation between configurations of 3000 combined updates. To each gauge configuration, 500 SD gauge fixings, starting from different randomly chosen points, were performed requiring

$$
\theta=\frac{1}{V N_{c}} \sum_{x} \operatorname{Tr}\left[\Delta(x) \Delta^{\dagger}(x)\right]=\frac{1}{V N_{c}} \sum_{x}|\partial \cdot A|^{2}<10^{-15} .
$$

From these $500 \mathrm{SD}$, on each gauge orbit, we keep the gauge configurations associated to the largest maximum of $F_{U}$ (named MAX in the following), the smallest maximum of $F_{U}$ (named MIN) and three random values of $F_{U}$ (RND1, RND2, RND3), generated starting the gauge fixing process by choosing always the same random $g(x)$ matrices. A further gauge fixing (named ID), starting the gauge fixing process by setting all $g(x)=1$, was performed to all gauge configurations. Another gauge fixing (named RND), starting the gauge fixing process by choosing always the same random $g(x)$ matrices, was performed to all configurations.

\subsection{Bare gluon propagator}

The scalar function $D\left(q^{2}\right)$, computed according to Eqs. (10) and (11), after averaging over equivalent momenta, ${ }^{3}$ is shown in Fig. 1 as function of $\hat{q}$ and as function of $q$. The figures include $D\left(q^{2}\right)$ as function of momenta of type $(q, 0,0,0),(q, q, 0,0),(q, q, q, 0)$ and $(q, q, q, q)$ for all available $q$ in our lattice. The figures for $D\left(q^{2}\right)$ are reported in Table 1. From now on, unless stated clearly, we will consider only the data referring to $D\left(q^{2}\right)$ as function of $q$.

Fig. 1 and Table 1 show that, for the gluon propagator, the effect of Gribov copies is small and visible for the smallest momenta. Indeed, comparing the different gluon propagators to the MAX propagator, it comes that, within one standard deviation, the

\footnotetext{
3 For example, for each gauge configuration the quoted value for momenta $(1,0,0,0)$ is the average over $(1,0,0,0),(0,1,0,0),(0,0,1,0)$ and $(0,0,0,1)$ values. Similarly, for $(1,1,0,0)$ a $Z_{4}$ average is performed, etc.
} 


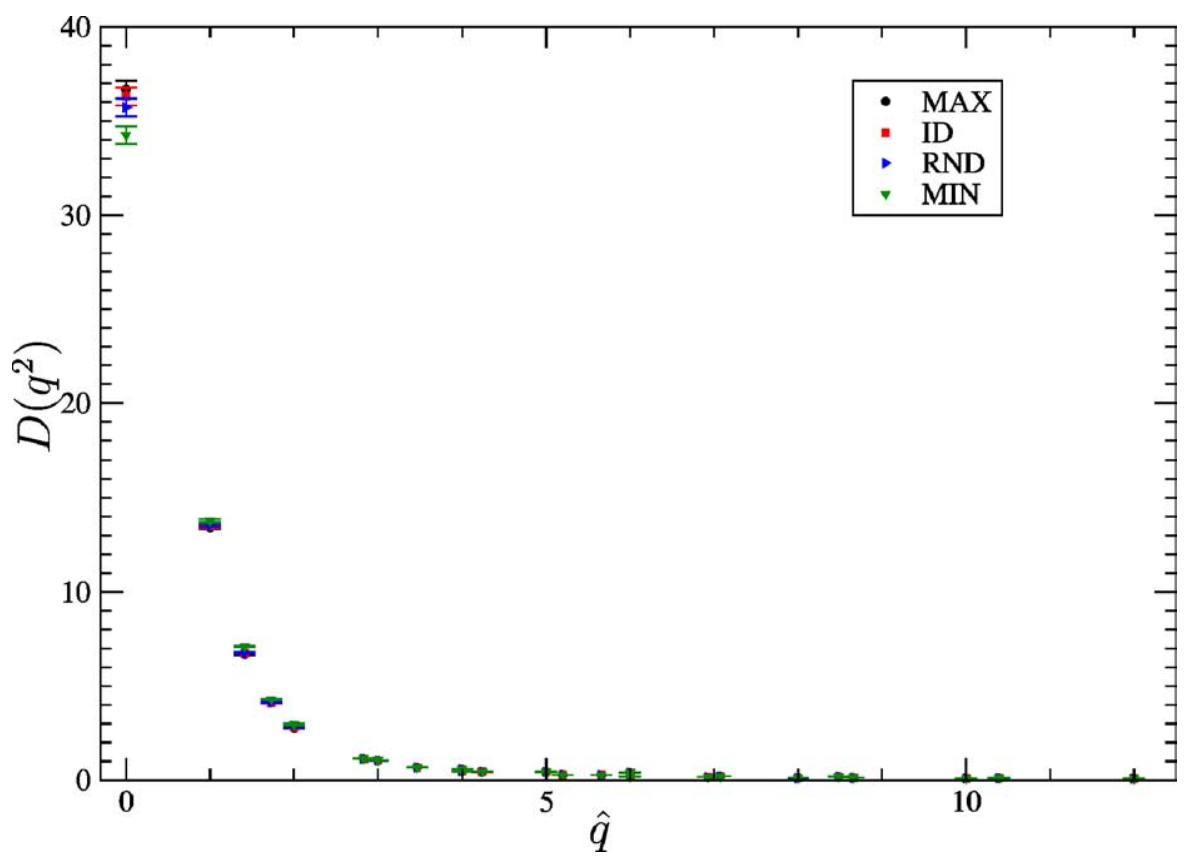

(a)

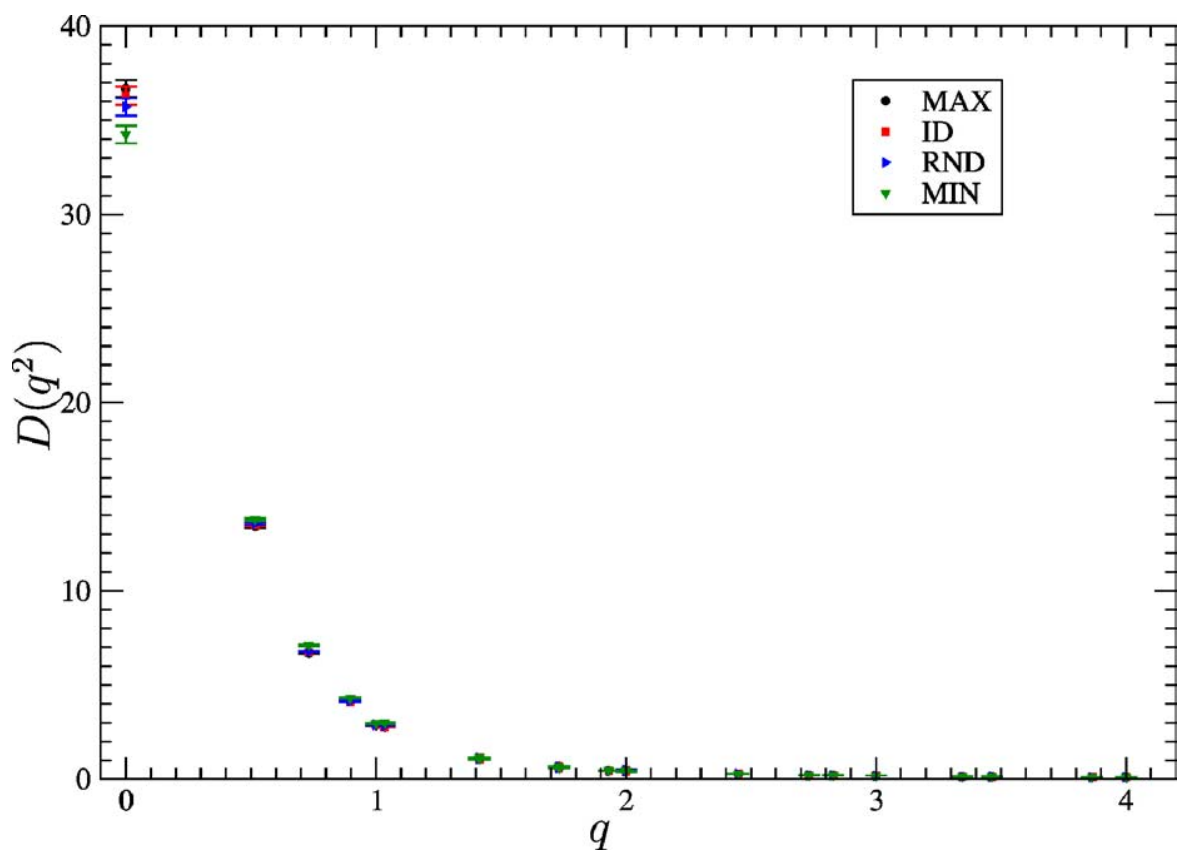

(b)

Fig. 1. Bare gluon propagator. Statistical errors were computed using the jackknife procedure. (a) Scalar function as function of $\hat{q}$. (b) Scalar function as function of $q$. 
Table 1

Bare gluon scalar function. The numbers in parentheses are the statistical errors, computed using the jackknife procedure, on the last digits of $D\left(q^{2}\right)$

\begin{tabular}{|c|c|c|c|c|c|}
\hline \multirow[t]{2}{*}{$n_{\mu}$} & \multirow[t]{2}{*}{$|q|$} & \multicolumn{4}{|l|}{$D\left(q^{2}\right)$} \\
\hline & & MAX & ID & RND & MIN \\
\hline$(0,0,0,0)$ & 0.0000 & $36.68(46)$ & $36.30(49)$ & $35.71(47)$ & $34.23(47)$ \\
\hline$(1,0,0,0)$ & 0.5176 & $13.436(79)$ & $13.496(79)$ & $13.556(75)$ & $13.780(77)$ \\
\hline$(2,0,0,0)$ & 1.0000 & $2.848(18)$ & $2.873(17)$ & 2.881(19) & $2.955(17)$ \\
\hline$(3,0,0,0)$ & 1.4142 & $1.0363(64)$ & $1.0397(64)$ & $1.0415(63)$ & $1.0566(61)$ \\
\hline$(4,0,0,0)$ & 1.7320 & $0.5769(34)$ & $0.5772(30)$ & $0.5771(34)$ & $0.5767(34)$ \\
\hline$(5,0,0,0)$ & 1.9319 & $0.4278(24)$ & $0.4280(25)$ & $0.4293(24)$ & $0.4316(25)$ \\
\hline$(6,0,0,0)$ & 2.0000 & $0.3892(32)$ & $0.3868(32)$ & $0.3878(30)$ & $0.3840(32)$ \\
\hline$(1,1,0,0)$ & 0.7320 & $6.693(32)$ & $6.752(35)$ & $6.760(35)$ & $7.100(38)$ \\
\hline$(2,2,0,0)$ & 1.4142 & $1.1303(61)$ & $1.1266(61)$ & $1.1349(56)$ & $1.1422(55)$ \\
\hline$(3,3,0,0)$ & 2.0000 & $0.4377(20)$ & $0.4390(20)$ & $0.4398(21)$ & $0.4401(21)$ \\
\hline$(4,4,0,0)$ & 2.4495 & $0.2635(13)$ & $0.2636(12)$ & $0.2637(13)$ & $0.2646(13)$ \\
\hline$(5,5,0,0)$ & 2.7320 & $0.2026(10)$ & $0.2020(10)$ & $0.2024(10)$ & $0.2019(10)$ \\
\hline$(6,6,0,0)$ & 2.8284 & $0.1866(13)$ & $0.1863(12)$ & $0.1867(13)$ & $0.1859(12)$ \\
\hline$(1,1,1,0)$ & 0.8966 & $4.123(27)$ & $4.109(26)$ & $4.131(27)$ & $4.295(27)$ \\
\hline$(2,2,2,0)$ & 1.7320 & $0.6725(41)$ & $0.6737(44)$ & $0.6693(41)$ & $0.6736(42)$ \\
\hline$(3,3,3,0)$ & 2.4495 & $0.2734(16)$ & $0.2747(16)$ & $0.2723(16)$ & $0.2761(16)$ \\
\hline$(4,4,4,0)$ & 3.0000 & $0.1681(10)$ & $0.1688(10)$ & $0.1692(10)$ & $0.1709(10)$ \\
\hline$(5,5,5,0)$ & 3.3461 & $0.13105(75)$ & $0.13264(75)$ & $0.13147(74)$ & $0.13156(79)$ \\
\hline$(6,6,6,0)$ & 3.4641 & $0.1216(10)$ & $0.1222(10)$ & $0.1208(10)$ & $0.1230(11)$ \\
\hline$(1,1,1,1)$ & 1.0353 & $2.775(33)$ & $2.795(33)$ & $2.831(34)$ & $2.972(38)$ \\
\hline$(2,2,2,2)$ & 2.0000 & $0.4674(53)$ & $0.4664(56)$ & $0.4730(54)$ & $0.4674(56)$ \\
\hline$(3,3,3,3)$ & 2.8284 & $0.2018(25)$ & $0.1993(25)$ & $0.1995(25)$ & $0.1967(24)$ \\
\hline$(4,4,4,4)$ & 3.4641 & $0.1238(16)$ & $0.1233(15)$ & $0.1228(16)$ & $0.1251(15)$ \\
\hline$(5,5,5,5)$ & 3.8637 & $0.0982(12)$ & $0.0972(12)$ & $0.0981(10)$ & $0.0965(11)$ \\
\hline$(6,6,6,6)$ & 4.0000 & $0.0894(14)$ & $0.0904(15)$ & $0.0899(15)$ & $0.0898(14)$ \\
\hline
\end{tabular}

ID propagator agrees with the $D\left(q^{2}\right)$ MAX for almost all the momenta considered. The exception being $D\left(q^{2}\right)$ for the momenta associated to $n_{\mu}=(5,5,5,0)$, compatible with the MAX value only within two standard deviations. Note that only the ID and MAX values agree for the infrared regime. The RND propagator agrees, within one standard deviation, with the MAX propagator for all momenta but the zero momenta. The zero momenta RND propagator agrees with the MAX $D(0)$ only within two standard deviations. The strongest deviation from the MAX propagator occurs when $D\left(q^{2}\right)$ is computed using the smallest of the maxima of $F_{U}$. The MIN propagator agrees, within one standard deviation, with MAX for momenta $|q| \geqslant 1.7320$ for momenta of type $(q, 0,0,0),|q| \geqslant 2.000$ for $(q, q, 0,0),|q| \geqslant 3.3461$ for momenta $(q, q, q, 0)$ and $|q| \geqslant 3.4641$ for $(q, q, q, q)$ momenta. For smaller momenta the differences between the $D\left(q^{2}\right)$ values can achieve six standard deviations. Indeed, the agreement between the MIN and MAX values quoted in the table are: six standard deviations for $n_{\mu}=(1,1,0,0)$; four standard deviations for $n_{\mu}=(1,1,1,0)$ and $(2,0,0,0)$; three standard deviations for $n_{\mu}=(0,0,0,0),(1,0,0,0)$ 


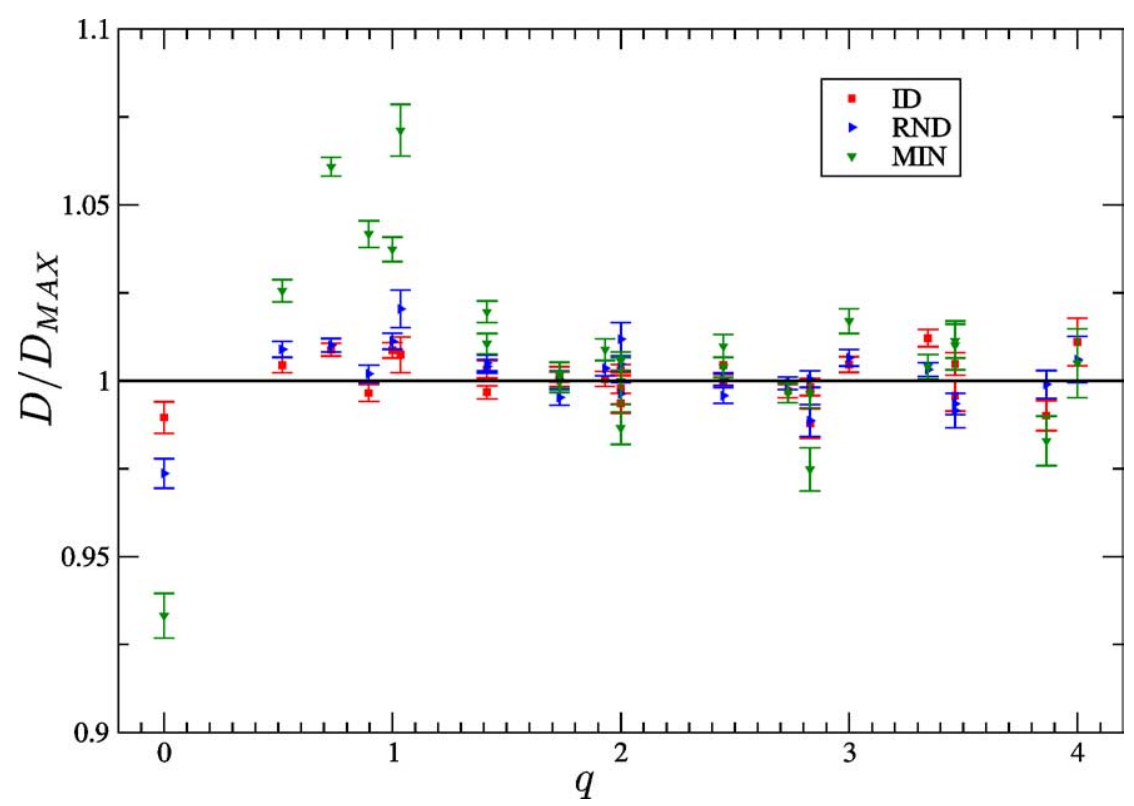

Fig. 2. $D\left(q^{2}\right) / D_{\text {MAX }}\left(q^{2}\right)$ as function of $q$ for ID, RND and MIN propagators. Statistical errors were computed using the bootstrap method. The quoted errors correspond to a $68 \%$ confidence limit of the distributions obtained from 5000 bootstrap samples.

and $(1,1,1,1)$; two standard deviations for $n_{\mu}=(2,2,0,0),(3,0,0,0),(3,3,3,3)$ and $(4,4,4,0)$. The lattice data shows clearly that Gribov copies change the low momenta $(q<1.7320)$ components of the gluon propagator.

For zero momentum, the largest propagator occurs when the configurations are gauge fixed to the fundamental modular region. The absolute difference between the MIN, RND and ID to the MAX zero momenta propagator central values are $6.7 \%, 2.6 \%$ and $1 \%$, respectively. These numbers can be read as an order of magnitude of the maximal change on the gluon propagator due to Gribov copies. For the other momenta, it is not always true that the largest value of $D\left(q^{2}\right)$ is associated to the MAX propagator. This can be seen in Fig. 2.

Fig. 2 suggests that the ratio between the propagators to the MAX propagator is a function of $q$, that converges to one for the larger momenta. Moreover, the figure shows clearly that the MIN propagator is different from the MAX propagator for momenta smaller than $q \sim 1.7$. From Fig. 2 one can quantify again the change on the gluon propagator due to Gribov copies. For the MIN propagator, the effect of Gribov copies is, at most, a factor of $5-10 \%$. For the RND and ID propagators, the effect of Gribov copies is not so dramatic (a factor smaller than $5 \%$ ).

Fig. 2 could suggest that the ratio between the propagators to the MAX propagator would be a constant factor. To test this hypothesis, in Fig. 3 the propagators are plotted after rescaling the different gluon fields to reproduce the central value of the zero momenta 


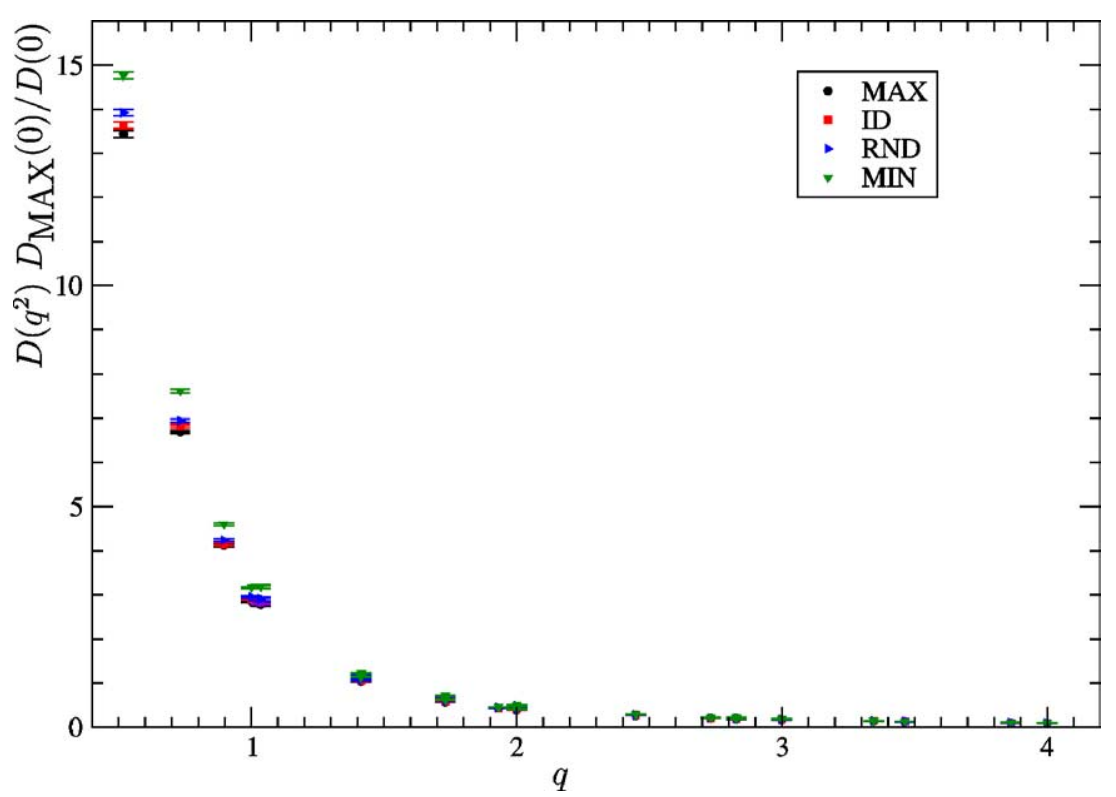

(a)

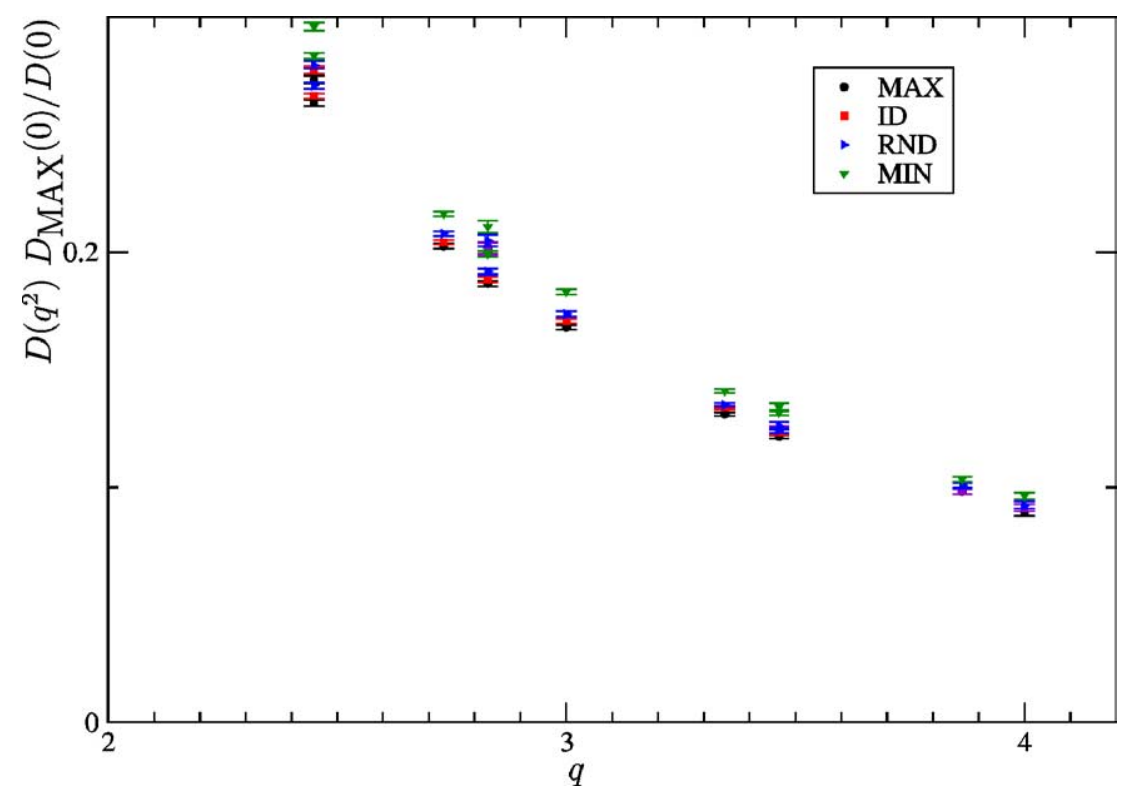

(b)

Fig. 3. Scaled gluon propagator. Statistical errors were computed using the jackknife procedure. (a) $D\left(q^{2}\right) D_{\mathrm{MAX}}(0) / D(0)$ as function of $q$ for all momenta. (b) $D\left(q^{2}\right) D_{\mathrm{MAX}}(0) / D(0)$ as function of $q$ for the larger momenta. 


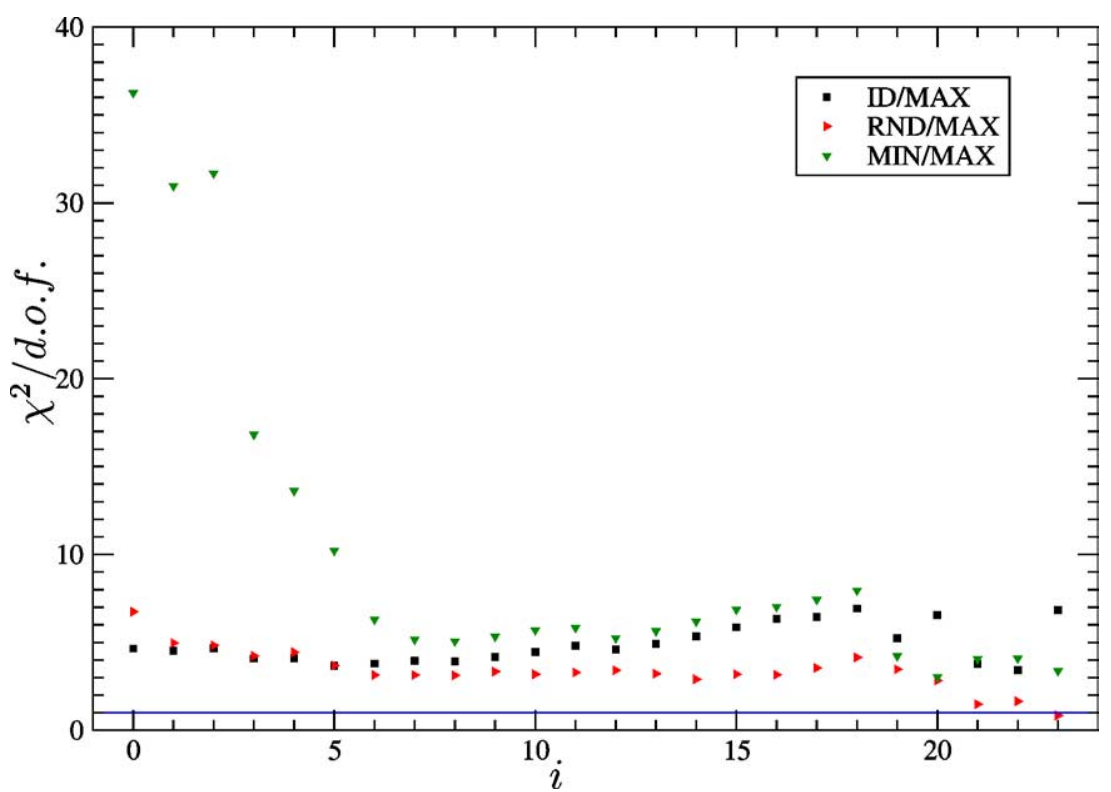

Fig. 4. $\chi^{2} /$ d.o.f. for the uncorrelated fits of $D\left(q^{2}\right) / D_{\mathrm{MAX}}\left(q^{2}\right)$ to a constant. $i$ is the number of lower momenta not considered in the fit.

MAX scalar function. As seen in Fig. 3, the propagators differ by more than one standard deviation for certain momenta. If, instead of rescaling the gluon field to reproduce the zero momenta MAX scalar function, the matching is done, for example, for $n_{\mu}=(6,0,0,0)$, then $D(0)=36.68 \pm 0.46,36.53 \pm 0.50,35.84 \pm 0.47$ and $34.70 \pm 0.48$ for the MAX, ID, RND and MIN propagators, respectively. The MAX, ID and RND $D(0)$ are compatible within one standard deviation. The MIN $D(0)$ agrees with MAX value only within three standard deviations. In order to further test the hypothesis under discussion, $D / D_{\mathrm{MAX}}$ was fitted to a constant. No correlations were considered in the fits. The $\chi^{2} /$ d.o.f. for these fits are reported in Fig. 4. Although, in general, the values of the $\chi^{2} /$ d.o.f. decrease as one excludes more lower momenta, they are always too high to conclude that the ID, RND and MIN propagators differ, from the MAX propagator, by a multiplicative factor. In particular, the difference between MIN, ID and MAX propagators is clearly not a constant. The RND/MAX ratio is compatible with a constant for the largest momenta considered.

In conclusion, the analysis of the raw data for the bare gluon propagator suggests that the effect of Gribov copies is small, but observable (clearly, less than a $10 \%$ factor) and is stronger for smaller momenta. Moreover, Gribov copies have almost no effect on the high momentum components of the gluon propagator. The data reported in Table 1 shows that the effect of Gribov copies can be overcame if one multiplies the statistical errors by a factor of 2 to 3 for the smaller momenta $(a q \leqslant 1.73)$. This doubling of the statistical error can be either, a general property associated to the effect of Gribov copies, or a result due to the limited statistics used here. Note that in the $S U(2)$ study of [16], the number of configurations used for the larger lattices $\left(12^{4}, 16^{4}\right)$ and for the larger $\beta(=2.7)$ was about half or less than half of the configurations used in our simulation. The investigation 
of $D\left(q^{2}\right) / D_{\mathrm{MAX}}\left(q^{2}\right)$ shows that the propagators associated to the Gribov copies named as ID, RND and MIN differ from the MAX propagator by more than a constant factor.

\subsection{Gribov copies and gluon propagator models}

In the previous section, it was argued that the ID, RND and MIN propagators do not differ from the MAX propagator by a constant factor. The question we would like to investigate now being: is it possible to quantify the differences, due to Gribov copies, when modelling the gluon propagator? To try to answer this question, we will study the fit of $D\left(q^{2}\right)$ to a functional form.

In [30] a number of gluon propagator models were studied. Our simulation access a limited range of momenta and, certainly, finite space and/or finite volume effects are no negligible. Instead of performing a detailed study of several functional forms, we chose to investigate the model which, according to Leinweber et al. [30], describes better the lattice data.

Let us assume that the scalar function is given by

$$
D\left(q^{2}\right)=Z\left[\frac{A M^{2 \alpha}}{\left(q^{2}+M^{2}\right)^{1+\alpha}}+\frac{L\left(q^{2}, M^{2}\right)}{q^{2}+M^{2}}\right],
$$

where

$$
L\left(q^{2}, M^{2}\right)=\left[\frac{1}{2} \ln \left[\left(q^{2}+M^{2}\right)\left(q^{-2}+M^{-2}\right)\right]\right]^{-d_{D}}
$$

is an infrared-regulated version of the one-loop logarithm correction to the gluon propagator and, for pure gauge theories, $d_{D}=13 / 22$.

According to the results of the previous section, Gribov copies seem to change the gluon propagator for the low energy momenta. Therefore, to measure such an effect we will consider three different types of uncorrelated fits. A fit to the highest momenta (UVfit) using the following functional form

$$
D\left(q^{2}\right)=\frac{Z}{q^{2}}\left\{\frac{1}{2} \ln \left(\frac{q^{2}}{\Lambda^{2}}\right)\right\}^{-d_{D}},
$$

a one-loop corrected perturbative gluon propagator. A fit to the lowest momenta (IR-fit), assuming that

$$
D\left(q^{2}\right)=\frac{A M^{2 \alpha}}{\left(q^{2}+M^{2}\right)^{1+\alpha}}
$$

and a fit of (30) to all lattice data.

In order to compare our results with [30], we take their central values for $a^{-1}$ at $\beta=6.0$ and $\beta=6.2$ and scale $a$ to $\beta=5.8$ using the results of two-loop calculations. This procedure gives, respectively, $a^{-1}=1.463 \mathrm{GeV}$ and $a^{-1}=1.590 \mathrm{GeV}$. The average of the two values being $a^{-1}=1.53 \pm 0.06 \mathrm{GeV}(a=0.13 \mathrm{fm})$.

Fig. 5 shows $q^{2} D\left(q^{2}\right)$ as function of $q$ for all sets of gauge fixed configurations. The results for the different momenta shows that, in our simulation, the finite space/volume 


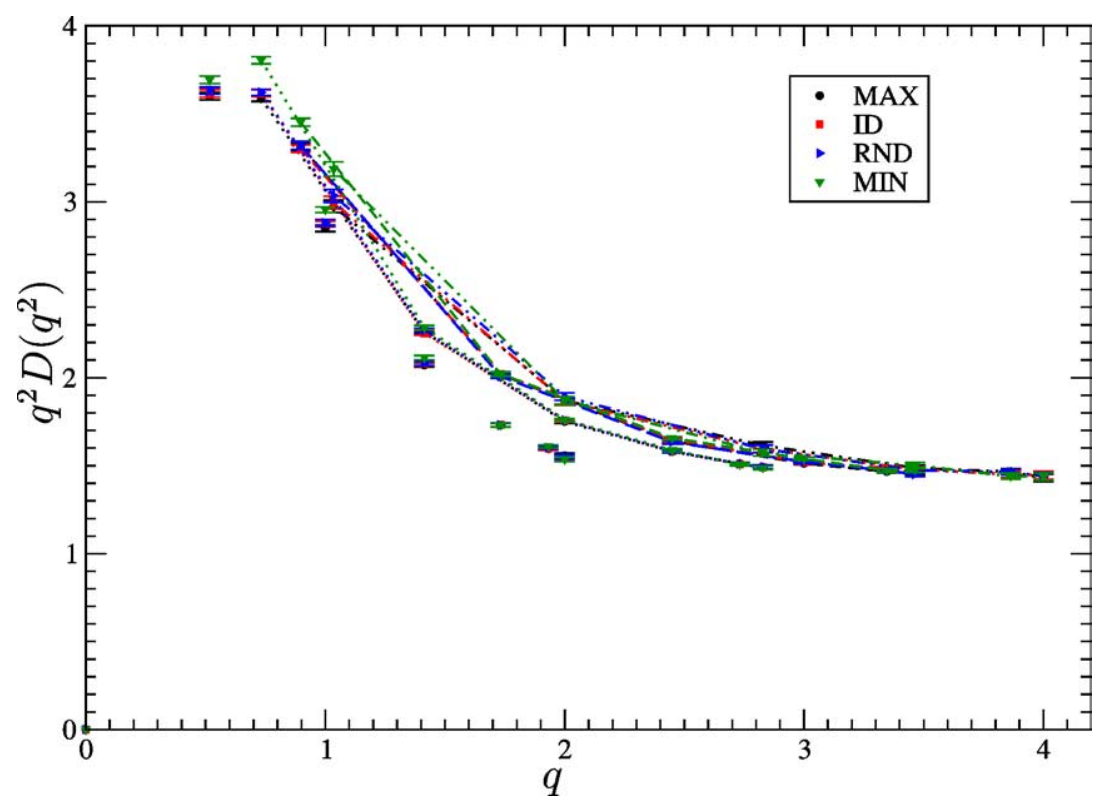

Fig. 5. $q^{2} D\left(q^{2}\right)$ as function of $q$ for MAX, ID, RND and MIN propagators. The points not connected by lines refer to $(q, 0,0,0)$ momenta. The points connected by dotted lines refer to $(q, q, 0,0)$ momenta, the points connected by dashed lines to $(q, q, q, 0)$ momenta and the points connected by dashed-dotted lines to $(q, q, q, q)$ momenta. Statistical errors were computed using the jackknife procedure.

Table 2

Fits of the higher momenta of type $n_{\mu}=(n, 0,0,0)$ to the one-loop corrected perturbative gluon propagator (32). The fitting range goes from $n=3$ to $n=6$. For larger fitting ranges, the $\chi^{2} /$ d.o.f. becomes too large $(>18)$. It is possible to fit the data using a smaller fitting range $(n=4$ to $n=6)$. However, we do not report the figures because such a fit would have only one degree of freedom. Statistical errors were computed using the bootstrap method. The quoted errors correspond to a $68 \%$ confidence limit of the distributions obtained from 5000 bootstrap samples

\begin{tabular}{llll}
\hline & $Z$ & $\Lambda$ & $\chi^{2} /$ d.o.f. \\
\hline MAX & $1.473_{-11}^{+11}$ & $0.8076_{-69}^{+71}$ & 0.08 \\
ID & $1.4578_{-98}^{+98}$ & $0.8181_{-65}^{+65}$ & 0.48 \\
RND & $1.4620_{-98}^{+97}$ & $0.8167_{-67}^{+66}$ & 0.44 \\
MIN & $1.4243_{-98}^{+98}$ & $0.8465_{-62}^{+66}$ & 2.78 \\
\hline
\end{tabular}

effects are not negligible - an effect of the order of $10 \%$ from $(q, 0,0,0)$ to the other types of momenta. Since the different types of momenta have different finite space/volume effects, we will not include different types of momenta in the fits. The exception being the IR fits.

The fits of the highest momenta to the asymptotic form (32) are reported in Tables 2-5 for all types of momenta. The first point to remark is that the gluon propagator scales perturbatively for $a q \geqslant \sqrt{2}$ for momenta associated to $n_{\mu}=(n, 0,0,0)$ and $n_{\mu}=$ $(n, n, 0,0)$, for $a q \geqslant 2.450$ for $n_{\mu}=(n, n, n, 0)$ momenta and for $a q \geqslant 1.035$ for $n_{\mu}=$ $(n, n, n, n)$ momenta; i.e., the asymptotic form describes quite well the lattice data for 
Table 3

Fits of the higher momenta of type $n_{\mu}=(n, n, 0,0)$ to the one-loop corrected perturbative gluon propagator (32). The fitting range goes from $n=2$ or 3 up to $n=6$. Statistical errors were computed using the bootstrap method. The quoted errors correspond to a $68 \%$ confidence limit of the distributions obtained from 5000 bootstrap samples

\begin{tabular}{|c|c|c|c|c|c|c|}
\hline & \multicolumn{3}{|c|}{$n_{\mu}=(2,2,0,0)$} & \multicolumn{3}{|c|}{$n_{\mu}=(3,3,0,0)$} \\
\hline & $Z$ & $\Lambda$ & $\overline{\chi^{2} / \text { d.o.f. }}$ & $Z$ & $\Lambda$ & $\chi^{2} /$ d.o.f. \\
\hline MAX & $1.7846_{-67}^{+61}$ & $0.7208_{-41}^{+46}$ & 1.44 & $1.861_{-19}^{+19}$ & $0.659_{-14}^{+15}$ & 0.14 \\
\hline ID & $1.7867_{-64}^{+51}$ & $0.7184_{-35}^{+45}$ & 0.44 & $1.823_{-17}^{+17}$ & $0.688_{-13}^{+13}$ & 0.15 \\
\hline RND & $1.7776_{-59}^{+57}$ & $0.7285_{-38}^{+41}$ & 0.82 & $1.829_{-17}^{+18}$ & $0.686_{-14}^{+13}$ & 0.27 \\
\hline MIN & $1.7617_{-55}^{+56}$ & $0.7413_{-37}^{+39}$ & 0.52 & $1.804_{-17}^{+15}$ & $0.706_{-12}^{+14}$ & 0.72 \\
\hline
\end{tabular}

\section{Table 4}

Fits of the higher momenta of type $n_{\mu}=(n, n, n, 0)$ to the one-loop corrected perturbative gluon propagator (32). The fitting range goes from 3 up to $n=6$. For larger fitting ranges, the $\chi^{2} /$ d.o.f. becomes too large $(>2)$. It is possible to fit the data using a smaller fitting range $(n=4$ to $n=6)$. However, we do not report the figures because such a fit would have only one degree of freedom. Statistical errors were computed using the bootstrap method. The quoted errors correspond to a $68 \%$ confidence limit of the distributions obtained from 5000 bootstrap samples

\begin{tabular}{llll}
\hline & $Z$ & $\Lambda$ & $\chi^{2} /$ d.o.f. \\
\hline MAX & $2.100_{-36}^{+37}$ & $0.534_{-27}^{+27}$ & 0.78 \\
ID & $2.165_{-40}^{+35}$ & $0.498_{-24}^{+27}$ & 1.29 \\
RND & $2.150_{-39}^{+40}$ & $0.499_{-27}^{+27}$ & 0.09 \\
MIN & $2.092_{-33}^{+36}$ & $0.555_{-27}^{+25}$ & 0.80 \\
\hline
\end{tabular}

Table 5

Fits of the higher momenta of type $n_{\mu}=(n, n, n, n)$ to the one-loop corrected perturbative gluon propagator (32). The fitting range goes from $n=1$ or 2 up to $n=6$. Statistical errors were computed using the bootstrap method. The quoted errors correspond to a $68 \%$ confidence limit of the distributions obtained from 5000 bootstrap samples

\begin{tabular}{|c|c|c|c|c|c|c|}
\hline & \multicolumn{3}{|c|}{$n_{\mu}=(1,1,1,1)$} & \multicolumn{3}{|c|}{$n_{\mu}=(2,2,2,2)$} \\
\hline & $Z$ & $\Lambda$ & $\overline{\chi^{2} / \text { d.o.f. }}$ & $Z$ & $\Lambda$ & $\chi^{2} /$ d.o.f. \\
\hline MAX & $2.099_{-11}^{+9}$ & $0.5944_{-45}^{+47}$ & 0.39 & $2.112_{-31}^{+27}$ & $0.584_{-19}^{+22}$ & 0.51 \\
\hline ID & $2.076_{-11}^{+8}$ & $0.6043_{-41}^{+50}$ & 0.52 & $2.102_{-31}^{+27}$ & $0.584_{-20}^{+24}$ & 0.64 \\
\hline RND & $2.0756_{-97}^{+79}$ & $0.6122_{-42}^{+48}$ & 1.12 & $2.064_{-28}^{+26}$ & $0.621_{-20}^{+22}$ & 1.48 \\
\hline MIN & $2.0146_{-93}^{+72}$ & $0.6516_{-41}^{+46}$ & 1.20 & $2.091_{-30}^{+31}$ & $0.590_{-23}^{+22}$ & 1.05 \\
\hline
\end{tabular}

sufficiently large momenta. Perturbative scaling starts at momenta $q \sim 1.6-3.7 \mathrm{GeV}$, a value compatible with the figure quoted in [30], $2.7 \mathrm{GeV}$.

In what concerns the effect of Gribov copies at high momenta, the results given in Tables 2-4 show that, for the same data and fitting range, the MAX, ID and RND values are compatible within one standard deviation. For momenta associated to $n_{\mu}=(n, n, n, n)$ 
and for the largest fitting range, ${ }^{4}$ the $Z$ and $\Lambda$ values are compatible within two standard deviations. On the other side, the MIN fitted parameters are not compatible with the MAX figures; the exception being the fit to $(q, q, q, 0)$ momenta and the fit to the smallest fitting range reported in Table 5.

In what concerns the stability of results, in general, the fitted parameters are not stable against a change in the fitting range. Probably, this is due to the limited number of different momenta available in the simulation. If one compares the results of the larger fitting ranges where $Z$ and $\Lambda$ are compatible within one standard deviation for the different types of momenta, it comes that $Z$ increases and $\Lambda$ decreases as one goes from $n_{\mu}=(n, 0,0,0)$ to $n_{\mu}=(n, n, n, n)$ by a factor of $\sim 1$.4. Such a large correction is an indication of important finite space effects-remember that the lattice spacing is $\sim 0.13 \mathrm{fm}$. If one compares our values for $\Lambda$ with those reported in [30], the numbers given in Tables 2-5 are, typically, larger than those reported by Leinweber et al.

The discussion of the IR properties of the gluon propagator requires data for small momenta. In our simulation one has only a limited access to the infrared regime of QCD. This is a serious limitation to a proper investigation of the low energy gluon propagator. Nevertheless, we have tried to find the combination of the smaller momenta which is well reproduced by (33). Unfortunately, to achieve such a goal, we had to combine different types of momenta. Below, we will show the results of such investigation. The reader should be aware of the physical meaning, or lack of meaning, of the numbers reported here. We would like to remember that our main goal is to see if there are differences, on the propagators, due to the choice of Gribov copies.

The set of momenta associated ${ }^{5}$ to $n_{\mu}=(0,0,0,0),(1,0,0,0),(1,1,0,0)$ and $(2,0,0,0)$ is well described by the model function (33). The fitted parameters are reported in Table 6 for the different propagators. Although, the lattice data is well described by (33), not all fitted parameters are compatible within one standard deviation. Indeed, the MIN propagator values are not compatible with any of the other propagators. The MAX and ID propagators all have the same $A$ parameter. The $A$ from the RND fit is, within two standard deviations, compatible with the MAX figures. In what concerns the gluon mass $M$, the MAX and ID values are compatible within one standard deviation but MAX and RND are compatible within three standard deviations. For the parameter $\alpha$, the MAX and ID values are compatible within one standard deviation but MAX and RND are compatible within two standard deviations. Note that the gluon mass $M$ computed from the IR regime of QCD is not compatible, within one standard deviation, with the values of $\Lambda$ from the UV regime-see Tables 2-5. The values of $M$ and $\alpha$ for MAX are the smallest figures in Table 6. From these fittings, one can quantify the effect due to Gribov copies as a two to three sigma effect on the parameters.

Finally, let us discuss the fittings of (30) to all lattice data. The results of the fits are reported in Table 7 for momenta $n_{\mu}=(n, 0,0,0)$, in Table 8 for $n_{\mu}=(n, n, 0,0)$, in Table 9 for $n_{\mu}=(n, n, n, 0)$ and in Table 10 for $n_{\mu}=(n, n, n, n)$ momenta. The $\chi^{2} /$ d.o.f. shows that, in general, the lattice data is well described by (30). The exceptions are the fits

\footnotetext{
${ }^{4}$ In physical units, the fitting range includes momenta from $1.6 \mathrm{GeV}$ up to $6.1 \mathrm{GeV}$.

${ }^{5} q=0,0.52,0.73$ and 1 or, in physical units, $q=0,0.80,1.12$ and $1.53 \mathrm{GeV}$, respectively. Note that the number of degrees of freedom for this fit is one.
} 
Table 6

The infrared propagator. Statistical errors were computed using the bootstrap method. The quoted errors correspond to a $68 \%$ confidence limit of the distributions obtained from 5000 bootstrap samples

\begin{tabular}{lllll}
\hline & $A$ & $M$ & $\alpha$ & $\chi^{2} /$ d.o.f. \\
\hline MAX & $17.72_{-24}^{+26}$ & $0.6947_{-59}^{+62}$ & $1.278_{-21}^{+23}$ & 0.048 \\
ID & $18.19_{-27}^{+29}$ & $0.7076_{-68}^{+67}$ & $1.312_{-24}^{+25}$ & 0.031 \\
RND & $18.78_{-27}^{+29}$ & $0.7237_{-64}^{+68}$ & $1.363_{-24}^{+26}$ & 1.032 \\
MIN & $22.81_{-44}^{+43}$ & $0.8189_{-96}^{+94}$ & $1.675_{-38}^{+37}$ & 1.561 \\
\hline
\end{tabular}

Table 7

Fits to all lattice data for momenta associated to $n_{\mu}=(n, 0,0,0)$ to the functional form (30). Statistical errors were computed using the bootstrap method. The quoted errors correspond to a $68 \%$ confidence limit of the distributions obtained from 5000 bootstrap samples

\begin{tabular}{llllll}
\hline & $Z$ & $A$ & $M$ & $\alpha$ & $\chi^{2} /$ d.o.f. \\
\hline MAX & $1.581_{-13}^{+11}$ & $12.63_{-28}^{+29}$ & $0.737_{-12}^{+12}$ & $1.982_{-56}^{+56}$ & 0.22 \\
ID & $1.564_{-11}^{+11}$ & $13.04_{-27}^{+30}$ & $0.748_{-11}^{+11}$ & $2.004_{-52}^{+52}$ & 0.39 \\
RND & $1.580_{-10}^{+10}$ & $13.82_{-27}^{+30}$ & $0.780_{-11}^{+11}$ & $2.134_{-48}^{+52}$ & 0.74 \\
MIN & $1.559_{-12}^{+09}$ & $15.69_{-32}^{+32}$ & $0.841_{-12}^{+12}$ & $2.320_{-56}^{+54}$ & 3.45 \\
\hline
\end{tabular}

to the MIN data, momenta $n_{\mu}=(n, 0,0,0)$, and ID propagator, momenta $n_{\mu}=(n, n, n, 0)$. For these two cases the $\chi^{2} /$ d.o.f. is quite large, meaning that the lattice data is not described by (30).

To identify the effect of Gribov copies the different fits are compared for the same type of momenta. The data on Tables 7-10 shows that, for all types of momenta, the fitted parameters for the MIN propagator are not compatible with the corresponding parameters for the MAX propagator. For momenta associated to $n_{\mu}=(n, 0,0,0)$, the ID and MAX propagators parameters are compatible within one standard deviation. The RND and MAX $Z$ values are compatible, within the same level of precision, the $\alpha$ and $M$ values are compatible within $2 \sigma$ and $A$ is compatible within three standard deviations. For $n_{\mu}=(n, n, 0,0)$ momenta, ID and RND parameters are compatible with the MAX values only within two standard deviations. The exception being the $\alpha$ from RND propagator, which agrees with the MAX figures within $1 \sigma$. For $n_{\mu}=(n, n, n, 0)$, RND and MAX values are compatible within two standard deviations. For the ID parameters, the $Z$ value is, within two standard deviation, compatible with the MAX value and all remaining parameters are compatible within $1 \sigma$. For $n_{\mu}=(n, n, n, n)$, the MAX, RND and ID fitted parameters are compatible within one standard deviation; the $Z$ for the ID and MAX are compatible within $2 \sigma$. Note that, in general, the MAX propagator has the larger $Z$ value and the smallest $A, M$ and $\alpha$. Again, like in the IR fits one can quantify the effect due to Gribov copies as a two to three sigma effect. From the fittings it is not possible to establish, clearly, which parameters are less sensible to Gribov copies. Note that the fits to $n_{\mu}=(n, n, n, n)$ momenta, although having large statistical errors and with the exception of the MIN propagator, they do not distinguish the Gribov copies. 
Table 8

Fits to all lattice data for momenta associated to $n_{\mu}=(n, n, 0,0)$, with $n$ from 0 to 6 , to the functional form (30). Statistical errors were computed using the bootstrap method. The quoted errors correspond to a $68 \%$ confidence limit of the distributions obtained from 5000 bootstrap samples

\begin{tabular}{llllll}
\hline & $Z$ & $A$ & $M$ & $\alpha$ & $\chi^{2} /$ d.o.f. \\
\hline MAX & $1.8565_{-41}^{+34}$ & $10.46_{-18}^{+20}$ & $0.7283_{-72}^{+76}$ & $1.990_{-31}^{+32}$ & 1.15 \\
ID & $1.8478_{-39}^{+33}$ & $11.02_{-18}^{+18}$ & $0.7493_{-68}^{+72}$ & $2.061_{-29}^{+28}$ & 0.12 \\
RND & $1.8430_{-38}^{+31}$ & $10.96_{-18}^{+20}$ & $0.7524_{-70}^{+77}$ & $2.046_{-28}^{+30}$ & 0.45 \\
MIN & $1.8055_{-35}^{+32}$ & $13.94_{-22}^{+20}$ & $0.8569_{-78}^{+74}$ & $2.389_{-30}^{+29}$ & 0.33 \\
\hline
\end{tabular}

Table 9

Fits to all lattice data for momenta associated to $n_{\mu}=(n, n, n, 0)$, with $n$ from 0 to 6 , to the functional form (30). Statistical errors were computed using the bootstrap method. The quoted errors correspond to a $68 \%$ confidence limit of the distributions obtained from 5000 bootstrap samples

\begin{tabular}{llllll}
\hline & $Z$ & $A$ & $M$ & $\alpha$ & $\chi^{2} /$ d.o.f. \\
\hline MAX & $1.9410_{-48}^{+36}$ & $10.26_{-27}^{+27}$ & $0.7371_{-91}^{+94}$ & $2.018_{-40}^{+39}$ & 1.71 \\
ID & $1.9535_{-47}^{+36}$ & $10.40_{-25}^{+28}$ & $0.7484_{-89}^{+97}$ & $2.071_{-39}^{+41}$ & 2.46 \\
RND & $1.9289_{-45}^{+40}$ & $11.05_{-29}^{+29}$ & $0.773_{-10}^{+10}$ & $2.144_{-42}^{+41}$ & 1.60 \\
MIN & $1.9090_{-42}^{+36}$ & $12.83_{-26}^{+27}$ & $0.8460_{-91}^{+95}$ & $2.355_{-38}^{+37}$ & 0.68 \\
\hline
\end{tabular}

Table 10

Fits to all lattice data for momenta associated to $n_{\mu}=(n, n, n, n)$, with $n$ from 0 to 6 , to the functional form (30). Statistical errors were computed using the bootstrap method. The quoted errors correspond to a $68 \%$ confidence limit of the distributions obtained from 5000 bootstrap samples

\begin{tabular}{llllll}
\hline & $Z$ & $A$ & $M$ & $\alpha$ & $\chi^{2} /$ d.o.f. \\
\hline MAX & $2.018_{-13}^{+12}$ & $10.38_{-81}^{+73}$ & $0.756_{-28}^{+24}$ & $2.15_{-12}^{+10}$ & 0.48 \\
ID & $1.993_{-13}^{+11}$ & $11.07_{-78}^{+75}$ & $0.780_{-26}^{+24}$ & $2.22_{-11}^{+10}$ & 0.44 \\
RND & $2.001_{-12}^{+10}$ & $10.42_{-66}^{+72}$ & $0.764_{-23}^{+24}$ & $2.117_{-95}^{+98}$ & 1.12 \\
MIN & $1.9239_{-87}^{+70}$ & $14.30_{-69}^{+74}$ & $0.896_{-20}^{+21}$ & $2.535_{-86}^{+89}$ & 0.57 \\
\hline
\end{tabular}

Table 11

\begin{tabular}{lllll}
\hline & $(q, 0,0,0)$ & $(q, q, 0,0)$ & $(q, q, q, 0)$ & $(q, q, q, q)$ \\
\hline$\alpha$ & $1.982_{-56}^{+56}$ & $1.990_{-31}^{+32}$ & $2.018_{-40}^{+39}$ & $2.15_{-12}^{+10}$ \\
$M(\mathrm{MeV})$ & $1128_{-18}^{+18} \pm 44$ & $1114_{-11}^{+12} \pm 44$ & $1128_{-14}^{+14} \pm 44$ & $1157_{-43}^{+37} \pm 45$ \\
\hline
\end{tabular}

From the analysis of Tables 7-10 one can check which parameters are robust against change of fitting momenta. Indeed, looking only to the fundamental modular region propagators, it comes that the overall normalization parameter $Z$ is a function of the type of momenta considered. At $1 \sigma$, the different $Z$ values are not compatible with each other. For 
the same level of precision, the $n_{\mu}=(n, n, 0,0), n_{\mu}=(n, n, n, 0)$ and $n_{\mu}=(n, n, n, n)$ fitted parameters which measures the relative normalization of the infrared to ultraviolet propagator components, $A$, are compatible with each other. On the other hand, $M$ and $\alpha$ parameters are robust against change of momenta. All four values reported in the tables are compatible within one standard deviation. Our results for $\alpha$ and $M$ are shown in Table 11 where the second error in $M$ represents the error in the lattice spacing. Curiously, these values are compatible, within one standard deviation with the values quoted in [30], namely $\alpha=2.2_{-0.2-0.3}^{+0.1+0.2}$ and $M=(1020 \pm 100 \pm 25) \mathrm{MeV}$. Note that the values for $M$ and $\alpha$ quoted for the fittings to all lattice data are not, in general, compatible with the same parameters computed from the IR and UV fits. Probably, this is due to using a relatively small lattice that does not enable a clear separation between the low energy and high energy regimes of QCD.

\section{Discussion and conclusions}

In this paper the problem of the Gribov copies in lattice QCD is addressed. To try to understand the role of Gribov copies in lattice QCD, the gluon propagator was computed with 302 configurations for a $12^{4}$ lattice and for $\beta=5.8$ using the overrelaxed quasi-heat bath.

The analysis of the raw data shows that Gribov copies change only the low momenta components of the gluon propagator. In our simulation, only for momenta $a q<1.7320$ $(q<2.6 \mathrm{GeV})$ there are significant differences between the MIN and MAX propagators. The RND data is not compatible, within one standard deviation, with the MAX propagator only for zero momentum. The study performed here shows that, typically, the choice of different Gribov copies changes the propagator in such a way that the figures become compatible within two-to-three standard deviations. Note, however, that for the pathological case of the MIN propagator the deviation relative to the MAX propagator, can be as large as six standard deviations effects. This result seems to suggest that in the study of the IR limit of the gluon propagator, the statistical errors should be multiplied by a factor of two or three in order to take into account possible deviations due to Gribov copies. If this is true for the statistical accuracy of our study, this may not hold when larger statistics, bigger lattices are considered. That the Gribov copies change essentially the IR limit of the propagator can be seen in Fig. 6, where $D\left(q^{2}\right)$ is plotted against $\left\langle F_{U}\right\rangle$.

The properties observed for the raw scalar data are observed when we model the lattice data. A difference, due to the choice of Gribov copies, of up to two-to-three standard deviations is seen on the fitted parameters. Note that this is observed even if the lowest energy momenta have the largest absolute errors, i.e., their contribution to the $\chi^{2}$ is not so relevant. To our mind, a deviation of this order of magnitude is, probably, a good measure of the influence of the Gribov copies on the gluon propagator.

In what concerns the gluon propagator itself, the results of our simulation for $M$ and $\alpha$ support the results quoted in a previous investigation using much larger lattices [30]. Remember that, for the MAX propagators, these parameters are robust against a change on the type of fitted momenta. It is interesting, that the lattice data supports quite well a gluon 


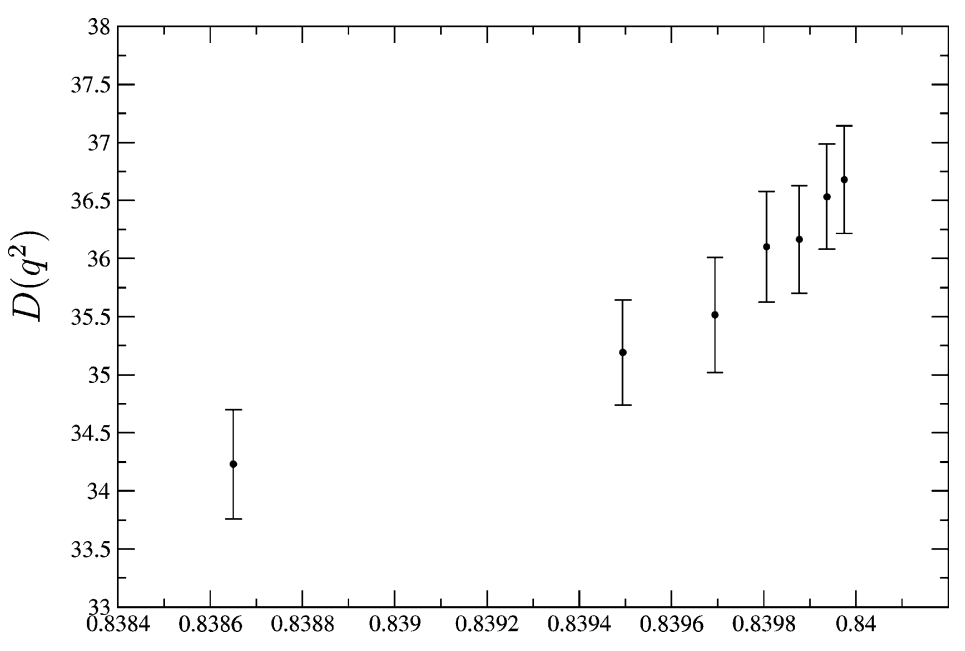

$\left\langle F_{U}\right\rangle$

(a)

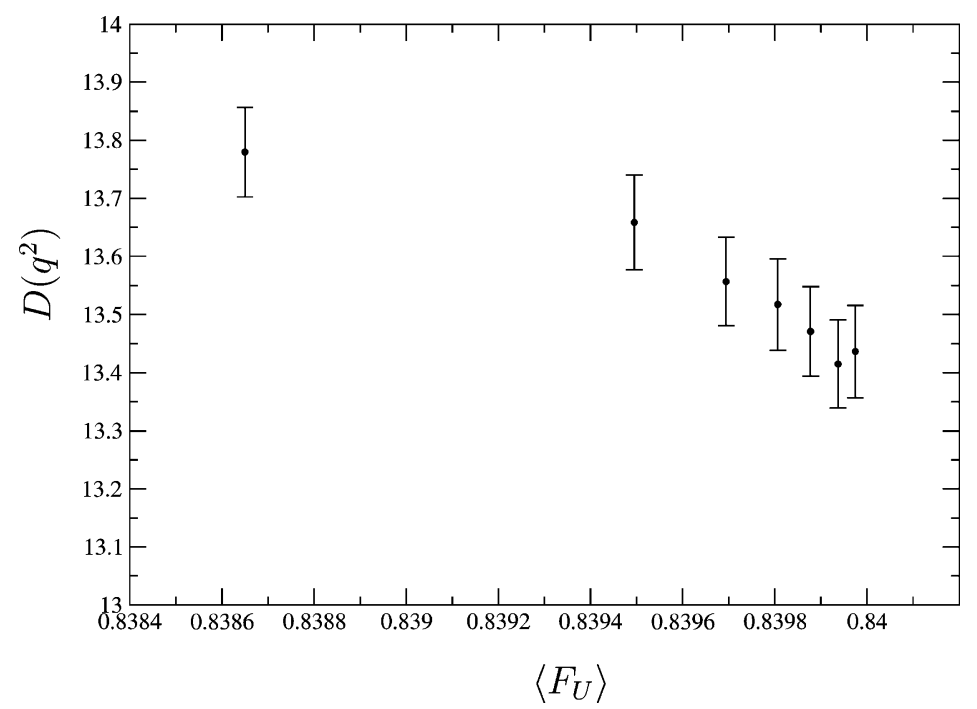

(b)

Fig. 6. Evolution of $D\left(q^{2}\right)$ with $\left\langle F_{U}\right\rangle$. Scalar function associated to (a) $n=(0,0,0,0)$; (b) $n=(1,0,0,0)$; (c) $n=(1,1,0,0)$; (d) $n=(1,1,1,0)$. After ordering the gauge fixed configurations associated with the sets MAX, ID, RND, MIN, RND1, RND2 and RND3 according to the $F_{U}$ value, the gluon scalar function is computed picking always configurations within the same class of values of $F_{U}$. Statistical errors were computed using the jackknife procedure.

propagator which, for large momenta, behaves like a massive vector with a mass of the order of the hadronic scale. Remember that a massive gluon propagator, with a gluonic mass of the order of $1 \mathrm{GeV}$, has some phenomenological support [31]. 


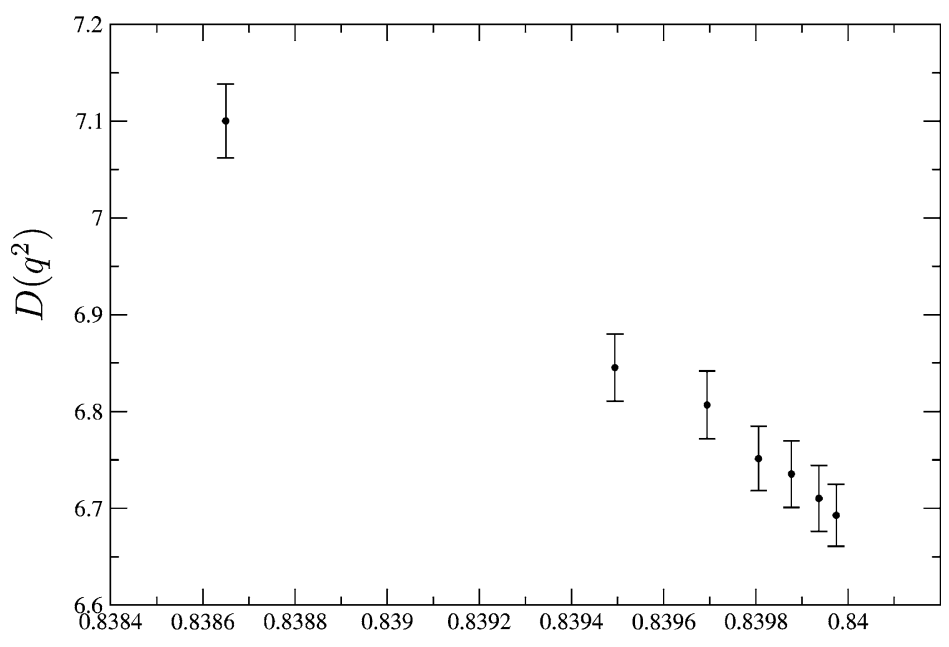

$\left\langle F_{U}\right\rangle$

(c)

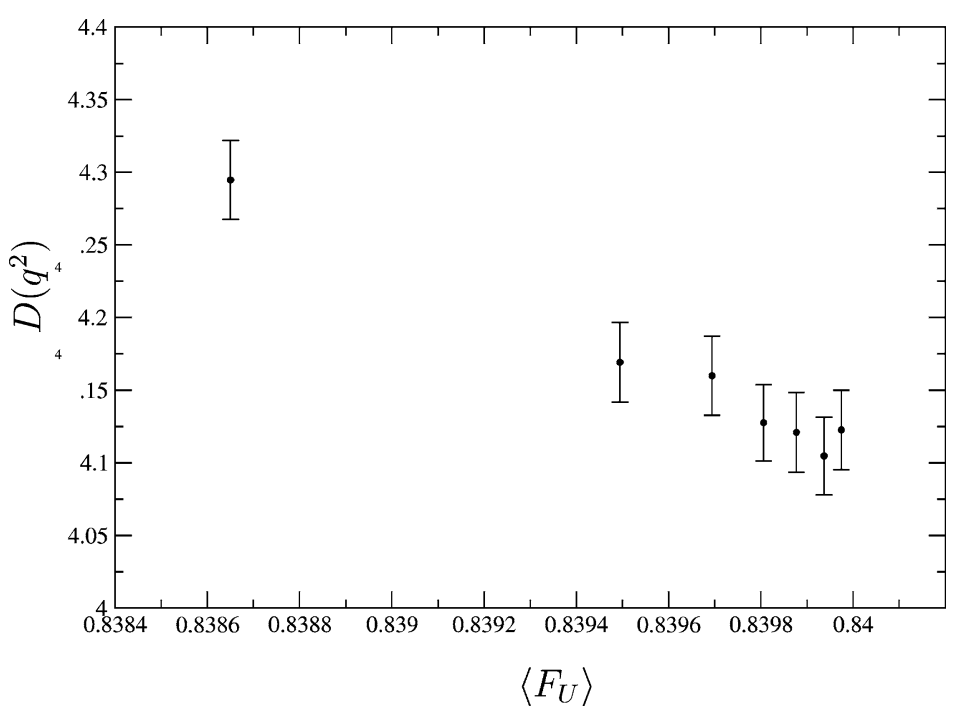

(d)

Fig. 6. (Continued).

\section{Acknowledgements}

P.J.S. acknowledges financial support from the Portuguese FCT. This work was supported by grant SFRH/BD/10740/2002. 


\section{References}

[1] R.P. Feynman, Acta Phys. Pol. 24 (1963) 262.

[2] B. DeWitt, Phys. Rev. 160 (1967) 113;

B. DeWitt, Phys. Rev. 162 (1967) 1195;

B. DeWitt, Phys. Rev. 162 (1967) 1293.

[3] L.P. Faddeev, V.N. Popov, Phys. Lett. B 25 (1967) 29.

[4] V.N. Gribov, Nucl. Phys. B 139 (1978) 1.

[5] S. Sciuto, Phys. Rep. 49 (1979) 181.

[6] I.M. Singer, Commun. Math. Phys. 60 (1978) 7.

[7] T.P. Killingback, Phys. Lett. B 138 (1983) 87.

[8] D. Zwanziger, Phys. Rev. D 69 (2004) 016002, hep-ph/0303028.

[9] A. Nakamura, R. Sinclair, Phys. Lett. B 243 (1990) 396.

[10] P. de Forcrand, Nucl. Phys. B (Proc. Suppl.) 20 (1991) 194.

[11] E. Marinari, C. Parrinello, R. Ricci, Nucl. Phys. B 362 (1991) 487.

[12] M.L. Paciello, C. Parrinello, S. Petrarca, B. Taglienti, A. Vladikas, Phys. Lett. B 289 (1992) 405.

[13] P. Marenzoni, P. Rossi, Phys. Lett. B 311 (1993) 219.

[14] M.L. Paciello, S. Petrarca, B. Taglienti, A. Vladikas, Nucl. Phys. B 341 (1994) 187.

[15] L. Giusti, M.C. Paciello, C. Parrinello, S. Petrarca, B. Taglienti, Int. J. Mod. Phys. A 16 (2001) 3487, heplat/0104012.

[16] A. Cucchieri, Nucl. Phys. B 508 (1997) 353, hep-lat/9705005.

[17] S. Furui, H. Nakajima, hep-lat/0309166.

[18] H. Nakajima, hep-lat/0309165.

[19] F. Semyonov-Tian-Shansky, in: Proc. Seminars of the Leningrad Math. Inst., 1982, Plenum, New York, 1986, English translation.

[20] G. Dell'Antonio, D. Zwanziger, Commun. Math. Phys. 138 (1991) 291.

[21] P. van Baal, Nucl. Phys. B 369 (1992) 259.

[22] P. van Baal, Nucl. Phys. B 417 (1994) 215.

[23] P. van Baal, hep-th/9511119.

[24] D. Zwanziger, Nucl. Phys. B 378 (1992) 525.

[25] D. Zwanziger, Nucl. Phys. B 412 (1994) 657.

[26] A. Cucchieri, Nucl. Phys. B 521 (1998) 365, hep-lat/9711024.

[27] C.H.T. Davies, G.G. Batrouni, G.P. Katz, A.S. Kronfeld, G.P. Lepage, P. Rossi, B. Svetitsky, K.G. Wilson, Phys. Rev. D 37 (1988) 1581.

[28] This work was in part based on the MILC Collaboration's public lattice gauge theory code. See http://physics.indiana.edu/ sg/milc.html .

[29] O. Oliveira, P.J. Silva, Comput. Phys. Commun. 158 (2004) 73, hep-lat/0309184.

[30] D.B. Leinweber, J.I. Skullerud, A.G. Williams, C. Parrinello, Phys. Rev. D 60 (1999) 094507, heplat/9811027;

D.B. Leinweber, J.I. Skullerud, A.G. Williams, C. Parrinello, Phys. Rev. D 61 (2000) 079901, Erratum.

[31] J.H. Field, Phys. Rev. D 60 (2002) 013013, hep-ph/0101158. 